\title{
La teoría económica y la epistemología
}

DOI: http://dx.doi.org/10.17981/econcuc.37.1.2016.01

Emmanuel Victorio Borgucci García ${ }^{1}$
Alberto Gregorio Castellano Montiel $^{2}$

\section{Resumen}

La ciencia económica ha recorrido un largo trayecto abundante de polémicas y críticas desde el punto de vista epistemológico y metodológico, concebido desde la época de Nassau W. Senior, John Stuart Mill y posteriormente con la disputa entre operacionalistas e instrumentalistas. Por lo que el objetivo de este estudio, parte de las consideraciones de la economía, realizadas por diferentes escuelas del pensamiento económico y se pone de relieve las dificultades que ha enfrentado la ciencia económica en su viaje a través de las diferentes posturas epistemológicas surgidas a lo largo de los siglos XIX y XX. En consecuencia, se realizó una revisión de los aportes principales que en materia epistemológica y metodológica han realizado las principales escuelas del pensamiento económico y de la epistemología general. Se concluye, que la economía moderna funciona más dentro de tradiciones de investigación que en escuelas de pensamiento, no busca la verificación completa y definitiva de sus proposiciones o la corroboración o falsación de sus teorías, pero si busca ser una ciencia realista perspectivista de corte naturalista.

Palabras clave: teoría económica; epistemología económica; metodología de la economía; tradiciones de investigación.

Recibido: 22.9.2015 Devuelto para revisión: 30.10.2015 Aceptado: 1.12.2015

\footnotetext{
${ }^{1}$ Doctor en Ciencias Sociales. Universidad Central de Venezuela (UCV). Magister en Banca y Finanzas, Economista. Universidad del Zulia. Profesor Titular de la Universidad del Zulia. Autor de correspondencia, eborgucci@yahoo.com

${ }^{2}$ Doctor en Ciencias Económicas. Universidad del Zulia (LUZ). Magíster en Gerencia de Empresas. Economista. Profesor titular de la Facultad de Ciencias Económicas y Sociales e Investigador adscrito al Instituto de Investigaciones de la Facultad de Ciencias Económicas y Sociales Universidad del Zulia (LUZ). albertocastellano@yahoo.com, castellanoalberto@fces.luz.edu.ve
}

The author; licensee Universidad de la Costa - CUC. 


\title{
The Economic Theory and Epistemology
}

\begin{abstract}
Economic science has reached to a long route abundant of controversy and criticism from the epistemological and methodological point of view, conceived from the time of Nassau W. Senior, John Stuart Mill and later with the dispute between operationalists and instrumentalists. The aim of this study, starts from the considerations of the economy, made by different schools of economic thought and highlights the difficulties that have faced economic science in its journey through the different positions posed epistemological throughout the nineteenth and twentieth centuries. Consequently, was made a review of the main contributions in epistemological and methodological matters that the main schools of economic thought were carried out and general epistemology. It is concluded that modern economy works more within research traditions than in schools of thought, it does not seek the complete and definitive verification of its propositions or corroboration or falsification of its theories, but if it seeks to be a realistic naturalist perspectivist science.
\end{abstract}

Keywords: economic theory; economic epistemology; methodology of the economy; research traditions.

\section{Introducción}

Desde el siglo XIX, los economistas más destacados de Europa y Estados Unidos han realizado intentos para hacer que la Economía posea plenamente un estatus de ciencia. No obstante, este anhelo ha encontrado innumerables dificultades y obstáculos tanto desde el punto de vista epistemológico como metodológico. Por esta razón, innumerables economistas a lo largo del tiempo han visua- lizado que el estudio de la Economía debe partir de supuestos y sus verdades deben ser válidas dentro de esos supuestos y en tanto la evidencia empírica lo respalde.

Producto de la postura deductivista impulsada principalmente por Nassau W. Senior, John S. Mill y, a mediados del siglo XX, por Milton Friedman, muchos economistas han empleado lo que ellos han denominado como condiciones, por ejemplo, de la preferencia 
del consumidor (Ferguson y Gould, 1979: 18); supuestos, por ejemplo, de conducta racional; principios como el del equilibrio del consumidor (Ferguson y Gould, 1979: 42); hipótesis como, por ejemplo, de las industrias de costos decrecientes (Zamora, 1977: 407) o hipótesis de las expectativas racionales (Hall y Taylor, 1988); leyes como las de demanda (Blaug, 1993); teoremas como el de Heckscher-Ohlin, o el teorema de Coase; o paradojas como las de Leontief o Giffen.

Un aspecto que se debe considerar en el discurso económico, según Schumpeter (1994), es que una proposición puede aparecer en una argumentación como postulado y en otra argumentación como teorema. Por otra parte, el mismo autor plantea que las hipótesis de la economía difieren de aquellas de las ciencias naturales no tanto por la carencia de resultados finales de alguna investigación, sino que son herramientas (en el sentido de Joan Robinson) elaboradas con la finalidad de respaldar un conjunto de proposiciones a priori.

Lo cierto es que el producto de la investigación científica en Economía es en muchos casos polémico y sujeto a toda serie de críticas. Autores como Bunge (1980) consideran que: "Quien se acerca a las ciencias sociales desde las ciencias naturales se siente inicialmente repelido por la oscuridad de la jerga, la pobreza e inexactitud de las ideas..."
(Bunge, 1980: 145). La ciencia económica como ciencia social, como dice Bunge (1980), está afectada, quizá más que las ciencias naturales, por las humanidades y la ideología. En primer lugar, porque la Filosofía otorga la visión del mundo, los problemas, las metodologías, las formas de conocimientos, la forma de obtención de resultados y su validez. En segundo lugar, las humanidades inciden sobre la ciencia económica por cuanto se ocupan de los problemas sociales, ya sea solucionándolos u ocultándolos.

Pero, como la Filosofía, como las ideologías políticas también polémicas en sus estudios y en los resultados de sus hallazgos, muchos economistas desestiman el uso de la metodología económica tal y como lo propone la Filosofía (Frey, 2000). Otra de las críticas a la Economía proviene de la metodología económica cuando, por ejemplo, a los datos se aplica la prueba " $t$ ", los resultados se presentan como si los datos hubiesen sido empleados una sola vez. Otra objeción surgió cuando McCloskey (1997) observó que un valor alto del estadístico " $t$ " asociado con un parámetro estimado es tomado para significar que la respectiva relación refleja una importante conexión empírica con el mundo real, cuando numerosos investigadores saben que tales inferencias son erradas. Por otra parte, McCloskey y Ziliak (1996) han señalado que en muchas investigaciones la interpretación de la idea de significancia estadística equiva- 
le a significancia substantiva, razón por la cual ambos autores se preguntan: ¿por qué entonces no prestan atención a los consejos de la metodología económica?

Finalmente, otra de las objeciones hacia la ciencia económica es que es una especie de retórica (McCloskey, 1983). Para McCloskey, la retórica es: "[e]l arte de probar lo que los hombres creen probando lo que es verdad de acuerdo a métodos abstractos" (1983: 482). Según esta definición, la retórica se convierte en un obstáculo epistemológico en el sentido de Bachelard (1997) y es dependiente de lo que McCloskey denomina como modernis$\mathrm{mo}^{3}$. Para McCloskey el ideal moderno es obsoleto, lo mismo que el neopositivismo lógico. Por esta razón, según este autor, la predicción en Economía es imposible, que se está haciendo uso de herramientas literarias y metáforas (mano invisible o capital humano)

\footnotetext{
${ }^{3}$ Cuyos preceptos en forma resumida son: 1) la predicción; 2) solamente las implicaciones observables de una teoría son consideradas como verdad; 3) la posibilidad de la observabilidad y los experimentos reproducibles; 4) si las implicaciones experimentales de una teoría prueban ser falsas, también lo es la teoría; 5) la observación subjetiva no es conocimiento científico; 6) el Dictum de Kelvin; 7) las creencias metafísicas o la introspección no forman parte de la justificación de proposiciones; 8) el papel de la metodología es demarcar las razones científicas de las no científicas; 9) la explicación científica de un evento lleva al evento bajo la cobertura de una determinada ley; 10) los científicos no tienen nada que decir acerca de los valores, sean estos morales o estéticos; y 11) el llamado "tenedor de Hume”.
}

para facilitar la recepción de la visión recibida. Esto ha conducido a la ciencia económica a debates y controversias como el surgido entre los keynesianos y monetaristas y que Blaug (1993) los califica: "como una de las controversias más frustrantes e irritantes de toda la historia del pensamiento económico..." (1993: 248).

Sin lugar a dudas, la Economía tiene mucho terreno que recorrer desde el punto de vista epistemológico y metodológico. En este sentido, partiendo de las consideraciones de la Economía realizadas por diferentes tradiciones de investigación o escuelas del pensamiento económico, y desde la perspectiva de la filosofía de la ciencia, este ensayo tiene como objetivo poner de relieve las dificultades que ha enfrentado la Economía en su viaje a través de las diferentes posturas epistemológicas surgidas en el siglo XIX y XX y dilucidar en cuál debería encajar de manera adecuada.

Ese tránsito no solamente ha sido problemático para la Economía sino para la epistemología de las ciencias naturales debido a que las diferentes posturas epistemológicas han evolucionado desde el neopositivismo lógico, el empirismo lógico o el falsacionismo, hasta arribar al debate entre el realismo científico y el antirrealismo. Así, se parte de que la Economía no está asociada al neopositivismo lógico pero sí lo está con el realismo. 
En consecuencia, el segundo objetivo es justificar la posibilidad que la ciencia económica se asocie a una forma de realismo científico de carácter disminuido y no asociado al instrumentalismo.

\section{Evolución de la Economía desde el punto de vista metodológico}

Según Blaug (1993), en el siglo XIX y comienzos del siglo XX, economistas principalmente británicos se dieron a la tarea de estudiar las posibilidades metodológicas de la ciencia económica en un contexto en que se estaban desarrollando un conjunto de avances dentro de la Filosofía de la Ciencia. Si bien, la Economía deseaba erigirse como ciencia en el sentido clásico del término, es interesante destacar que el análisis propuesto por los economistas se encontró con una serie de obstáculos. Ese grupo de pensadores de la ciencia económica estaba constituido inicialmente Adam Smith, David Ricardo, Nassau William Senior, John Stuart Mill, John Elliot Carines, John Neville Keynes y Lionel Robbins, Milton Friedman y Paul Samuelson entre otros.

Según John Neville Keynes (1990), Adam Smith empleó tanto el método a priori como el método a posteriori, debido a que no existe un método exclusivo de estudio. Según Keynes: "Él creyó en un orden natural de los eventos [que] pueden ser deducidos a priori de consideraciones de carácter general; pero él constantemente revisó sus resultados apelando el curso real de la historia" (1990: 11). Es decir, Smith partió de abstracciones y las confrontó con las realidades del mundo en que vivió, y propuso doctrinas de tendencias en donde combinaba esas consideraciones a priori con una investigación inductiva de las causas que restringen o confirman la operación de la tendencia propuesta.

Con relación a David Ricardo, según Rima (1995), los Principios de Economía Politica y Tributación fue una obra que se caracterizó porque: 1) no realizó la reflexión y revisión teórica- metodológica que sí efectuó Adam Smith, o como dice John Ferguson: "Ricardo, negociante labora como si viviese en un mundo de abstracciones" (1979: 102); y 2) como dice Rima: "[e]l estilo es abstracto en extremo y rara vez aligerado con digresiones sobre Historia y Filosofía como las encontramos en la obra de Smith" (1995: 146).

Para Nassau William, el papel del economista no es recomendar o disuadir, sino descubrir los principios generales, de acuerdo con la evidencia, relacionados con la generación y distribución de la riqueza. Además, según Senior, la economía política es solamente una ciencia que sirve a los propósitos del "arte del gobierno" (Senior, 1854:7). 
Para Senior, la ciencia de la economía política descansa en una serie de postulados que resultan de la observación y la conciencia. Estos postulados son: 1) "cada individuo desea obtener una riqueza adicional con el menor sacrificio posible" (1854: 34); 2) "que la población del mundo, o, en otras palabras, el número de personas que habitan en él, es limitado solamente por la moral o el demonio físico, o por el miedo a la deficiencia de esos artículos de la riqueza, el cual los hábitos de los individuos de cada clase de sus habitantes impelen a requerir" (1854: 34); 3) "que el poder del trabajo, y de los otros instrumentos con los cuales produce la riqueza, puede ser indefinidamente incrementado por medio del uso de sus productos como medios de mayor producción" (1854: 34); y 4) "que las habilidades en la agricultura permanecen iguales, la agregación de un número adicional de trabajadores empleados en la tierra en un determinado distrito produce en general un retorno proporcionalmente menor, o, en otras palabras, que a pesar del incremento del número de trabajadores empleados, el retorno agregado no es proporcional al incremento del número de trabajadores" (1854: 34).

John Stuart Mill, en su obra titulada Essays on Some Unsettled Questions of Political Economy (1874), deja claro lo siguiente respecto a lo que considera es la economía política: "[l]a ciencia que trata de la produc- ción y la distribución de la riqueza y de su dependencia de la leyes de la naturaleza humana". Desde el punto de vista metodológico, Mill caracterizó a la economía política como una ciencia abstracta y a su método como a priori, en donde la razón funciona no sobre hechos, sino sobre supuestos, y en este sentido expresa:

"el método a priori es un modo legítimo de investigación filosófica en las ciencias morales: nosotros agregamos que es el único modo. Podemos afirmar que el método $a$ posteriori o ese de la experiencia específica es no obstante ineficaz en esas ciencias como medio para llegar a un considerable cuerpo de verdades valiosas; sin embargo, se admite que este método es útil para ayudar al método a priori y aún forma un indispensable suplemento de éste" (1874: 15).

No obstante, Mill hacía algunas consideraciones adicionales: 1) cuando los principios de la economía política son aplicados a casos particulares, se deben tomar en consideración las circunstancias individuales de cada caso; 2) esas circunstancias son las que aparecen en todos los casos y las que no son comunes a todos los casos; 3) se pueden presentar circunstancias no comunes que representan factores perturbadores (disturbing causes), que representan un elemento de incertidumbre inherente a la naturaleza compleja del proceso económico; 4) la existencia de elementos perturbadores no impide 
el empleo del método a priori; 5) los elementos perturbadores tienen causas que siguen determinadas leyes y que pueden operar, en ocasiones, sobre la conducta humana, pero que pueden ser previstas a priori.

Para John Elliot Cairnes, toda relación que puede establecer el ser humano con la naturaleza consiste en acciones del primero sobre el segundo y de las impresiones que el segundo suscita en el primero. Por tanto, los efectos o los fenómenos dependen de dos causas, según Cairnes (2001: 26): de las propiedades que son inherentes a los objetos y las formas en que las propiedades de los objetos han actuado; por tanto, Cairnes propone el método inductivo. Su definición de inducción es similar a la de John Stuart Mill, veamos: "That operation of the Mind by which we infer that what we know to be true in a particular case or cases will be true in all cases which resemble the former in certain assignable respects" (Cairnes, 2001: 48) ${ }^{4}$. Como Cairnes propone el método de la inducción para estudiar los fenómenos de la producción y distribución de la riqueza, se debe comenzar por la observación y la experimentación. Pero debido a la complejidad de los fe-

${ }^{4}$ Es decir: la inducción es la operación de la mente por medio de la cual cuando algo es verdad en un caso particular, o en algunos casos, será verdad en todos los casos. Esta similitud se refiere, continua Cairnes, para determinados individuos de una clase o cuando algo es verdad para cierto periodo de tiempo. nómenos, el investigador se debe preguntar acerca de la naturaleza de los fenómenos y sus elementos accesorios para inferir leyes.

En la lectura V de The Character and Logical Method of Political Economy, Cairnes apunta que la Economía tiene grandes problemas para lograr altos niveles de precisión como, por ejemplo, la Química. Este grado de precisión, no es posible en la economía política. Por esta razón, para Cairnes, la economía política está excluida del dominio de las ciencias exactas, ya que la ley económica solo representa una verdad hipotética y no una positiva.

Con relación al marxismo, como materialismo dialéctico o método dialéctico, Bunge (1980) lo considera más como un enfoque. Según el autor, si bien se puede hablar de una ontología y metafísica dialéctica idealista o materialista, se trata de hipótesis muy generales acerca del devenir sociohistórico basado en la búsqueda de polaridades. Según Bunge, "[e]stas hipótesis más o menos precisas no constituyen un método, esto es, un procedimiento para hacer o estudiar algo. Constituyen una doctrina" (1980: 160). De acuerdo con lo antes expuesto, según Manuel Sacristán, para Marx "[l]a noción de ciencia económica coherente con ese enfoque del problema del método no es ni la noción de ciencia económica positiva hoy común, ni la noción de historia positiva hoy común, sino 
la noción dialéctica de una ciencia históricosocial sui ge-neris" (1978: 9)5 . Sacristán continua expresando: "Esa ciencia tiene su tipo propio de abstracción: aunque abstracción, abstracción histórica, que no se podría practicar más que sobre la base de un desarrollo (Entwicklung) determinado de la sociedad" (1978: 9).

Al considerar lo expresado por Sacristán, según Bunge (1980), concebir la realidad, y sobre todo la realidad social, de manera bipolar es una exageración, por cuanto la ciencia lo que le interesa es encontrar pautas (que generalmente se les denomina leyes) que no siempre son bipolares, ya que la realidad social y económica es más compleja de lo que aparenta. Es decir, el hecho de que la realidad económica sea dinámica no significa que sea en todo tiempo y lugar bipolar.

En el contexto de la preponderancia de los autores clásicos, surge el historicismo ale-

\footnotetext{
${ }^{5}$ Según Sacristán (1978), basado en un texto de Marx de 1857 (texto que no se publicó, la citada introducción de 1857 que se suele citar con las líneas fundamentales de la Crítica de la Economía Política- Grundrisse- y en el epílogo a la segunda edición del libro I de El Capital de 1873), expone: 1) un buen método de la Economía es ascender de lo abstracto a lo concreto; y 2) la explicación de la existencia de una correlación entre el orden lógico y ascendente de las categorías y su orden histórico. Con relación a este último punto su comentario es negativo, pues son órdenes inversos. De este modo, Sacristán concluye que el proyecto de Marx es buscar un conocimiento histórico que ha de servir al proyecto dialéctico.
}

mán de la vieja y nueva escuela histórica alemana, que según Schumpeter:

\begin{abstract}
"El artículo de fe básico y característico del método de la escuela histórica, dice que el órganon de la economía científica ha de consistir principalmente- y primero pensaron que exclusivamente- en los resultados de las monografías históricas y en sus generalizaciones. Por lo que hace a la parte científica de su vocación, el economista debe dominar antes que nada la técnica histórica. Con ayuda de esa técnica, que era todo el cuerpo científico que necesitaba, había que zambullirse en el océano de la historia económica. Para investigar esquemas o procesos concretos con todos sus detalles vivos, de lugar y tiempo y aprender a catar su bouquet. De este tipo de investigación ha de desarrollarse paulatinamente la única clase de conocimiento general accesible en ciencias sociales" (1994: 885).
\end{abstract}

Por su parte, la escuela nueva liderada por Gustav Schmoller enfatizaba que hay que dejar que los hechos económicos hablen por sí mismos de la inevitabilidad de las teorías económicas para dar cuenta de hechos históricos, el rechazo de las amplias generalizaciones de la filosofía de la historia, un rechazo del monismo metodológico y lo que Schumpeter denominó como el "error naturalista" (1994: 889) de Auguste Comte.

Pero la irrupción de la escuela histórica (Historische Schule deutscher Nationalöko- 
nomie) produjo una reacción en 1883 de parte de Carl Menger con su escrito Unterssuchungen über die Methode der Sozialwissenschaften in der Politischen Ökonomie insbesondere $^{6}$, que buscaba reivindicar las bondades del análisis teórico. Este trabajo fue reseñado negativamente en Zur Methodologie der Staats- und Sozialwiessenschaften $^{7}$ por parte de Gustav Schmoller en el Jahrbuch für Gesetzgebung ${ }^{8}$ y Menger reiteró su ataque a la escuela histórica con Die Irthumer des historismus in der deutschen Nationalökonomie ${ }^{9}$ de 1884 . Pero como dice Schumpeter, se fue reconociendo paulatinamente la necesidad del análisis histórico en la Economía y la necesidad de la teorización económica en el análisis históri$\mathrm{co}^{10}$. En su primer trabajo, Menger expresó lo siguiente:

\footnotetext{
${ }^{6}$ Investigaciones sobre el método de las ciencias sociales en la política económica en particular.

${ }^{7}$ Sobre la metodología del Estado y las ciencias sociales.

${ }^{8}$ Anuario de legislación.

${ }^{9}$ Los errores del historicismo en la economía nacional alemana.

${ }^{10}$ En este sentido es interesante destacar que la obra de Arthur Spiethoff, según Joseph Schumpeter, formaba parte de lo que él denominó como la novísima escuela histórica junto a Max Weber y Werner Sombart. Según Schumpeter (1994), para Spiethoff, las teorías se desarrollan mediante la convergencia de un aparato conceptual simple y la formulación de hipótesis provisionales que traten de dar cuenta de los fenómenos económicos.
}

"La Escuela histórica comete, pues, un error en lo que respecta a estos problemas teóricos. Por una parte, los concibe de un modo demasiado estrecho: pasa por alto otras divergencias que, en lo que respecta a la metodología de nuestra ciencia, tienen la misma importancia que aquellas a las que ha dirigido su exclusiva atención; por otra parte, sus seguidores creen erróneamente que las dificultades teóricas para la teoría económica derivadas del desarrollo de los fenómenos y de sus divergencias de un lugar a otro pueden eliminarse ampliamente a través del método histórico" (2006: 185).

En su segunda obra, Menger observa lo que él consideró como tres errores del historicismo alemán: 1) aplicarse sólo a los estudios histórico-estadísticos, dejando de lado el aspecto teórico de la Economía; 2) ignorar la distinción entre ciencias teóricas y ciencias prácticas de la economía política; y 3) solamente tomar en cuenta los aportes teóricos que están "en estrecha relación con los estudios histórico-estadísticos" (Menger, 2006: 335).

Para Henry Sidgwick (1901), la aproximación a los fenómenos económicos reclama un análisis de series históricas, pero eso no soslaya el hecho de que es necesario complementarlo con análisis de carácter deductivo. Es decir, la economía política no emplea como método único la deducción 
o la inducción: “... la teoría general de la Economía Política, como comúnmente se le conoce, usa principalmente un método deductivo e hipotético" (Sidgwick, 1901: 40); pero: "[e]s obvio, no obstante, que los resultados obtenidos no nos hacen por ellos mismos capaces para interpretar o predecir adecuadamente los fenómenos económicos, y que antes de que nuestras conclusiones puedan ser efectivamente aplicadas para esos propósitos, se requiere más conocimiento que sólo se puede obtener por medio de la inducción" (Sidgwick, 1901: 40). Por esta razón, Sidgwick consideró que la ciencia de la economía política debe tener una fuerte relación con la historia general.

En The Scope and Method of Political Economy (1990), John Neville Keynes definió a la Economía como un cuerpo de doctrina relacionada con el fenómeno económico, que implica las acciones humanas dirigidas directamente a la creación, apropiación, acumulación de riqueza, por la costumbre económica y las instituciones económicas relacionadas con la riqueza. En este sentido propuso lo siguiente: 1) la economía es tanto una disciplina positiva como normativa siguiendo a Nassau William Senior; 2) no todos los hechos económicos pueden ser aislados de otros hechos sociales; 3) el método a posteriori no es conveniente para la investigación en Eco- nomía; 4) el procedimiento correcto es el método a priori, que parte de algunos hechos fundamentales de la naturaleza humana; y 5) de acuerdo con lo anterior, el homo economicus de John Stuart Mill es una abstracción.

Con el desarrollo de las ideas neoclásicas, tanto en el Reino Unido como en Europa continental principalmente, se va legitimando progresivamente el empleo de la Matemática en la ciencia económica. Según Schumpeter: "[l]a novedad se produce cuando el razonamiento mismo que arroja el resultado es un razonamiento explícitamente matemático y en este sentido preciso conozco tres casos claros anteriores a von Thünen y Cournot: D. Bernoulli, Beccaria, y --si estamos dispuestos a dar importancia suficiente a un mero indicio de sistema de equilibrio-Isnard" (1994: 1041). En este sentido, según Schumpeter, algunas de las razones para la popularidad del empleo de la Matemática en Economía son: 1) su empleo en el análisis teórico, el cual es cuantitativo, pero no numérico; 2) altera la actitud del economista con respecto a los problemas que surgen de los esquemas teóricos; 3) surgen nuevos métodos de prueba y refutación; 4) se puede saber hacer más de las relaciones entre variables; 5) elimina muchas controversias; 7) el lenguaje matemático se puede traducir al no matemático o: "[l]a Matemática no añade 
nada a la Economía, nada que no se pueda descubrir sin ella" (1994: 1043) ${ }^{11}$.

\footnotetext{
11 Es muy interesante resaltar las opiniones de Mays (1962) con respecto a la obra de William Stanley Jevons con relación al empleo de las Matemáticas en Economía. Mays se refería a Notice of a General Mathematical Theory of Political Economy como una obra que marcó un antes y después en el análisis económico. Entre sus primeros críticos se encontraban: John Elliot Carines y Alfred Marshall, ya que la teoría del valor y su exposición por parte de Jevons se desmarcaba de la perspectiva clásica. Pero entre los personajes que brindaron un apoyo decisivo a Jevons, se encontraban el astrónomo y matemático George Howard Darwin (1845-1912), hijo del famoso naturalista Charles Darwin, y Leon Walras. Darwin fue el primer gran defensor de las propuestas de Jevons frente a los ataques de Cairnes y Walras; escribió una carta a Jevons en donde ambos compartieron opiniones similares acerca de sus teorías matemáticas del intercambio. Pero según Mays (1962), Walras no leyó Teoría de la Economía Política de Jevons, y gracias a Johann d `ulnis, un estudiante en la Universidad de Leiden, Países Bajos, y posteriormente profesor en la Universidad de Utrecht, al reconocer Walras la autoría primogénita del trabajo de Jevons, Walras, por medio de Joseph Garnier, logró que las correspondencias entre ambos autores (Inglaterra y Lausanne) se publicasen en el Journal des Economistes. El enfoque matemático de la Economía no había todavía logrado una penetración en Europa continental, debido a que Alemania era el reducto de la Escuela histórica. En el Reino Unido mientras tanto, el empleo de las técnicas matemáticas fueron ganando popularidad, toda vez que Jevons encontró que en la Sociedad Estadística de Manchester, en donde se desarrolló un trabajo acerca de la teoría matemática de la economía política, tuvo comentarios favorables de Alfred Marshall. Los progresos en el empleo de las técnicas matemáticas en Economía se acrecentaron con personajes de la talla de Francis Ysidro Edgeworth y Phillip H. Wicksteet. Un segundo trabajo de W. S. Jevons titulado On Study of Periodic Commercial Fluctuations, de 1882, es considerado por John Maynard Keynes (biógrafo de Jevons) como una obra que dio comienzo a una nueva era en la ciencia económica..." (Mays, 1962: 216).
}

Lionel Robbins, en su Ensayo sobre la naturaleza y significancia de la ciencia económica, afirmó que:

"La naturaleza de la teoría económica es clara: es el estudio de las consecuencias formales de estas relaciones entre fines y medios dados diversos supuestos relativos a la naturaleza de los datos reales" (1980: $65)$.

Conocido el contenido de la ciencia económica y el significado relativo de las magnitudes económicas, Robbins propone que se aboque a precisar la significación que debe atribuirse a los resultados logrados. Según Robbins, las proposiciones fundamentales de la economía son las provenientes de la teoría del valor (1980: 107). Estas proposiciones descansan:

“... en el supuesto de que las diferentes cosas que un individuo desea tener poseen para él una importancia diversa y pueden ser dispuestas, por consiguiente, en un orden determinado [...]. Pero, en último análisis, se reduce a que podemos juzgar si diferentes experiencias posibles son para nosotros de una importancia mayor, menor o equivalente [...]". (Robbins, 1980: 109 - 110).

De todo lo anterior concluye Robbins que la naturaleza del análisis económico consiste: 
“... en deducciones derivadas de una serie de postulados de los cuales los más importantes son hechos casi universales de la experiencia en todos los casos en que la actividad humana tiene un aspecto económico; el resto lo constituyen supuestos de naturaleza más limitada y descansan en las características generales de situaciones particulares o tipos de situaciones para cuya explicación ha de usarse la teoría" (1980: 138).

\section{La irrupción del instrumentalismo en el análisis económico}

La corriente metodológica conocida como instrumentalismo tuvo como alguno de sus máximos exponentes a Pierre Duhem y Bas van Frassen. El máximo exponente del instrumentalismo en la ciencia económica es Milton Friedman. Este autor plantea que no es posible asimilar la llamada economía positiva $^{12}$ con las ciencias físicas. Según Friedman:

\footnotetext{
${ }^{12}$ La idea que adoptó Friedman de economía positiva la debe a John Neville Keynes. En su libro The Scope and Method of Political Economy, escrito en 1891, la expone de la siguiente manera: "John Neville Keynes distingue entre «una ciencia positiva... [,] un cuerpo de conocimientos sistematizados referente a lo que es; una ciencia normativa o reguladora... [,] un cuerpo de conocimientos sistematizados que discute criterios respecto a los que debería ser...; un arte... [,] un sistema de reglas para la consideración de un fin determinado», y comenta que «la confusión entre ellas es común y ha sido el origen de muchos errores perjudiciales; y urge la importancia de "reconocer una ciencia positiva precisa de la Economía Política»" (Friedman, 1967: 9).
}

"La Economía, en cuanto a ciencia positiva, es un cuerpo de generalizaciones a título experimental acerca de los fenómenos económicos, que puede usarse para predecir las consecuencias de los cambios en las circunstancias. El progreso en la ampliación de este conjunto de generalizaciones, el fortalecimiento de nuestra confianza en su validez y el mejoramiento de la exactitud de las previsiones que suministra se ven obstaculizados no sólo por las limitaciones de la capacidad humana que impide cualquier investigación para la adquisición de conocimientos, sino también por los obstáculos que son especialmente importantes para las ciencias sociales en general y la economía en particular, aun cuando no sean peculiares a ellas" (1967: 41).

Además, Friedman plantea que el investigador en Economía debe aceptar el hecho de obtener conocimientos por medio de lo que denomina como "experiencia incontrolada", es decir: "el principio metodológico fundamental de que una hipótesis únicamente puede verificarse por la conformidad de sus implicaciones o predicciones con los fenómenos observables; pero hace que la tarea de verificar las hipótesis sea más difícil y proporcione mayores oportunidades de confusión acerca de los principios metodológicos implicados..." (1967: 42).

A lo anterior Friedman agrega que no puede verificarse una teoría comparando directamente sus supuestos con la reali- 
dad, y añade: "[e]n verdad no existe forma significativa alguna de llevar a cabo esta comparación. Un "realismo» completo es claramente inalcanzable, y el problema de si una teoría es lo "suficientemente» realista puede resolverse únicamente observando si suministra predicciones suficientemente buenas para el objetivo de que se trate o si son mejores que las ofrecidas por teorías alternativas" (1967: 42). En consecuencia, la propuesta de Friedman se le ha conocido como la tesis de la irrelevancia de los supuestos, que desde el punto de vista metodológico, según Munt y Barrionuevo significa:

“... que los economistas deben someter a prueba empírica sus teorías independientemente de verificar el realismo de los supuestos. En este marco, una teoría económica contará con la aceptación de la comunidad de economistas si, siendo sometida a falsación, no ha sido refutada y ha presentado una considerable capacidad predictiva" (2010: 5).

En otras palabras, las propuestas de Friedman se asemejan a las de Duhem (1969) cuando proponía que la ciencia se ocupa de establecer relaciones entre observaciones que no sólo exhiben apariencias sensibles mediante proposiciones que establezcan relaciones entre observaciones para obtener nuevas relaciones entre observaciones sin importar si son verdaderas o falsas, sino que sean instrumentos de predicción.
Pero esta visión también se asemeja a la postura de van Frassen cuando plantea que:

"Presentar una teoría es especificar una familia de estructuras, sus modelos; y en segundo lugar, especificar ciertas partes de esos modelos (las subestructuras empíricas) como candidatos para la representación directa de los fenómenos observables. Podemos llamar apariencias a las estructuras que pueden describirse en los informes experimentales y de medición. La teoría es empíricamente adecuada si tiene algún modelo tal que todas las apariencias son isomórficas con las subestructuras empíricas de ese modelo" (1996: 89).

Esto se conoce como adecuación empírica. Además, los modelos deben contar con fuerza lógica, lo cual, según van Frassen (1996), es simplemente cuando hay menos modelos que den cuenta de las apariencias de determinado modelo.

\section{El operacionalismo particular de Samuelson}

Según Mark Blaug, el operacionalismo: “[e] s la posición metodológica que considera que las teorías e hipótesis serán científicas si, y sólo si, es posible especificar una operación física que asigne valores cuantitativos a sus términos fundamentales" (1993: 
296). Desde esta perspectiva, Samuelson habla del "teorema operacionalmente significativo" cuando una hipótesis empírica puede ser aceptada o rechazada de manera real o ideal. Esta idea surgió debido a que Samuelson demostró que los supuestos asociados a un problema de maximización no son suficientes para derivar todas las predicciones que se hagan de algún objeto de estudio económico.

Samuelson cuestionaba la perspectiva instrumentalista de Friedman debido a que: 1) la ciencia económica se reduciría a establecer infinitas correlaciones y solamente serán admisibles aquellas que sean de interés del investigador; 2) debido a que existen fenómenos económicos difíciles de cuantificar, cómo se pueden establecer predicciones; 3) cuando se relacionan cantidades económicas para observar su comportamiento, esas relaciones se hacen desde algún punto de vista, ya sea intuitivo o elaborado. Sin embargo, según Blaug (1993), una definición de esta manera se aleja del concepto de operacionalismo de Bridgman y se acerca más al falsacionismo popperiano ${ }^{13}$.

\footnotetext{
${ }_{13}$ Cuando se habla de operacionalismo es necesaria la referencia a Percy Bridgman. Según Bridgman (1927), cuando se abordan las demostraciones de las teorías es necesario introducir definiciones explícitas y traducibles a términos observacionales por medio de las denominadas "reglas de correspondencia". La dificultad de establecer reglas de correspondencias explícitas llevó a Percy Bridgman a introducir la noción de conceptos operacionales.
}

En resumen, los grandes metodólogos en la ciencia económica del siglo XIX $\mathrm{y}$ comienzos del siglo XX centraron su atención en que: 1) los conceptos o definiciones económicas debían derivarse de la observación y la introspección; 2) una vez evaluadas las definiciones, asumirían el papel de supuestos a priori; 3) se procedería a relacionar esas premisas para obtener razonamientos que serían contrastados con los respectivos referentes empíricos para, que libres de perturbaciones, obtener premisas $a$ posteriori. Es decir, como dice Blaug (1993), no son premisas de las que se pueda esperar que sean verdaderas o falsas, sino en qué caso son proclives de ser aplicadas.

\section{La Economía y la epistemología}

En su intento de ser considerada una ciencia, los estudiosos de la Economía han tenido que enfrentar toda una serie de desafíos verdaderamente serios. En el camino, para bien o para mal, esos desafíos han transitado por una serie de transformaciones, al menos cuando se confrontan con las exigencias de, por ejemplo, el neopositivismo lógico del Círculo de Viena. En primer lugar, la Economía se enfrentó al desafío del criterio de reducción y de demarcación 
sobre la base del fisicalismo y la teoría de la verificación del significado inicialmente desarrollada por Wittgenstein (2003).

En primer lugar, "[s]i los enunciados elementales (o "enunciados protocolares" como los denominaban Otto Neurath y Rudolf Carnap) deben ser la base para los enunciados intersubjetivos de la ciencia, ellos mismos tendrían que ser intersubjetivos; tenían que referirse no a experiencias privadas, incomunicables, sino a acontecimientos físicos públicos" (Ayer, 1981: 25).

En segundo lugar, siguiendo a Hempel, se tiene que: "una oración $\mathrm{S}$ tiene significado empírico si, y sólo si, es posible indicar un conjunto de oraciones observacionales, O1, O2, ..., On; tales que, si fuesen verdaderas, también $\mathrm{S}$ lo sería necesariamente" (Hempel, 2006: 109 - 110).

En tercer lugar, se debe considerar el hecho de que las magnitudes económicas debían contar con definiciones explícitas y ser traducibles a términos observacionales por medio de los denominados "conceptos operacionales", que para Percy Bridgman: "[n]o es nada más que una serie de operaciones" (1927: 59). Esta postura fue criticada, por ejemplo por Carl Hempel y Karl Popper. Con Hempel, los conceptos operacionales "son especificaciones parciales de significado" (Hempel, 1981: 128). Para Karl Popper:

"Las mediciones presuponen teorías. No hay medición sin teoría y ninguna operación puede ser descrita satisfactoriamente en términos no teóricos. Los intentos de hacerlo contienen siempre un círculo vicioso; por ejemplo, la descripción de las mediciones de longitud requiere una teoría (rudimentaria) de las mediciones del calor y la temperatura; pero éstas implican, a su vez, mediciones de longitud" (1991: 90).

Además, el autor antes mencionado agrega que la introducción y especificación de los significados es relativa y formal (necesita el reconocimiento de la audiencia académica).

En cuarto lugar, la Economía debía producir teorías basadas en enunciados observacionales referidos a experiencias sometidas a una incontrastable verificación de los enunciados. Sin embargo, los enunciados económicos se encuentran con que los datos que sirven de base para la realización de las verificaciones presentan todo tipo de imprecisiones, y, que según Shackle, se expresa de la siguiente forma: 
"La Economía es la ciencia que, de entre todas las disciplinas académicas, salta con mayor atrevimiento el abismo que se abre entre las humanidades y las ciencias físicas. Haciendo virtud de la imprecisión que su doble cara le impone, ha desarrollado la técnica de los números índices hasta convertirla en una rama absorbente y sutil de las matemáticas prácticas" (1976: 378).

Es decir, en la Economía los enunciados observacionales se deben enfrentar a un problema de agregación más problemático que, por ejemplo, el de las ciencias naturales. La fuente de información de la Economía proviene de datos contables, que dependen de reglas de formación de, por ejemplo, estados financieros sometidos a reglas de construcción convencionales propias, por ejemplo, de la contabilidad general, y reprocesadas en términos de la contabilidad nacional.

Así, las escuelas de pensamiento económico debían enfrentar el problema de la agregación, de la precisión de las magnitudes teóricas y el problema del reconocimiento de una gran mayoría de la audiencia académica. El problema de manera concreta lo plantea Bunge (1989) cuando considera que: 1) no todas las magnitudes son definibles en un contexto dado, algunas deben tomarse como primitivas o no definidas (Bunge, 1989: 146); 2) para
Bunge, los científicos nunca calculan magnitudes, sino sus valores numéricos; 3) se debe buscar un modo adecuado para la introducción de conceptos que denotan propiedades y relaciones potenciales.

A lo anterior se debe añadir que autores como Toulmin (1953) cuestionaron la idea de que exista un lenguaje común a todas las teorías independiente de su grado de formalización. En el caso de Hanson (1972) se demuestra que sobre la misma imagen pueden verse objetos diferentes, debido a que dos personas observan en función de su experiencia visual y conceptual previa, es decir, no existe una base observacional común de las percepciones.

En quinto lugar, las proposiciones protocolares de la Economía debían verificarse de manera única y definitiva. El problema para la Economía es que sus enunciados y resultados no pueden ser extraídos de experimentos y mucho menos de experimentos únicos y definitivos.

Afortunadamente para la Economía, este problema se presentó en las ciencias naturales. La imposibilidad de la verificación como criterio único de significado y de demarcación entre enunciados, llevó a la idea de incremento gradual de la confirmación (Gradually increasing of confirma- 
tion) propuesta por Carnap (1992). Si la verificación es el medio para el completo y definitivo establecimiento de la verdad, entonces las proposiciones universales nunca podrán ser verificadas, incluidas las de la Economía. Sin embargo, pueden ser confirmadas por medio de la acumulación de la suficiente evidencia empírica.

A pesar que puede parecer como más adecuado generar investigación económica, como lo propone Carnap, se presenta el problema de la inducción, la teoría de la dependencia de las observaciones y el de las probabilidades, que no son de la misma naturaleza que en las ciencias naturales. En el caso de la Economía, se emplea lo que Popper denominó como probabilidades subjetivas y que: "[t]rata el grado de probabilidad como si fuese una medida de los sentimientos de certidumbre o incertidumbre, de creencia o de duda, que pueden surgir en nosotros ante ciertas aserciones o conjeturas" (Popper, 1980: 139). Dentro de las probabilidades subjetivas, este autor distinguió las probabilidades psicológicas de lo que denominó como probabilidades lógicas y cuyo máximo exponente es John Maynard Keynes, y que definió como: "[u]n tipo especial de relación lógica entre dos enunciados” (1980: 139).

En consecuencia, cómo inferir lógicamente la ocurrencia de un evento futu- ro sobre la base de experiencias acumuladas en el pasado. Sólo se podría decir que la ocurrencia y frecuencia de eventos es "probablemente verdadera". Posteriormente Popper dice:

"No existe nada que pueda llamarse inducción. Por tanto, será lógicamente inadmisible la inferencia de teorías a partir de enunciados singulares que estén verificados por la experiencia. Así pues, las teorías no son nunca verificables empíricamente. Si queremos evitar este error positivista de nuestro criterio de demarcación que elimine los sistemas teóricos de la ciencia natural, debemos elegir un criterio que nos permita admitir en el dominio de la ciencia empírica incluso enunciados que no pueden verificarse" (1980: 40).

Para Popper, el proceso científico comienza con la observación que supone la existencia de un sistema de teorías y preconcepciones existentes previamente. Por otra parte, el criterio de demarcación que propuso Popper no es el de la verificabilidad, sino el de la falsabilidad. Para Popper una teoría es falsada cuando: 1) se han aceptado enunciados básicos que la contradigan; 2) se produzca un "efecto reproducible" que refute a la teoría (Popper 1980: 83); es decir, una teoría es falsada cuando se corrobora una hipótesis empírica (hipótesis falsadora) de bajo nivel que 
describe un determinado estado de cosas o relaciones ${ }^{14}$.

Sin embargo, en Economía, la aplicación del falsacionismo presenta dificultades de las hipótesis ad hoc como, por ejemplo, con la función de consumo de John Maynard Keynes (1981). Esta función determina que el gasto de consumo ${ }^{15}$ depende del ingreso disponible ajustado por un coeficiente denominado propensión marginal al consumo, que en términos gráficos es la pendiente de la función de consumo e indica la proporción del ingreso disponible por unidad al consumo y al ahorro.

\footnotetext{
${ }^{14}$ El principio de falsación emplea como regla lógica el modus tollens, en donde: Si P, entonces Q. Q es falso. Entonces $\mathrm{P}$ es falso o $[(p \rightarrow q) \Lambda \sim q] \sim p$. Se presentan dos premisas y una conclusión. La primera premisa es una condición (hipótesis) y la segunda es una situación de falsedad (contrastación negativa). Se concluye que la premisa principal es falsa.

${ }^{15}$ Se debe aclarar que una cosa es consumo y otra cosa gasto de consumo. Esta diferencia se nota claramente cuando se habla de bienes durables, no durables y servicios. En el caso de los bienes durables, el gasto de consumo proviene cuando se financia la adquisición de un bien durable, mientras que su consumo ocurre en un periodo de tiempo relacionado con la vida útil del bien. En el caso de los bienes no durables y servicios, la distinción no es tan clara.
}

De esta manera $\mathrm{C}=\mathrm{bYd}^{16}$, donde $C$ es el gasto de consumo agregado, $b$ la propensión marginal al consumo o $b=\mathrm{dC} / \mathrm{dYd}$, y $Y d$ el ingreso disponible. Luego, el error se presenta cuando $\mathrm{E}=\mathrm{C}$-bYd, donde $E$ es el error. Entre 1970 y 1990, el comportamiento en el gasto del consumo generó errores negativos y positivos. Los errores negativos estuvieron asociados con una visión pesimista de los consumidores, alza del petróleo, etc., mientras que los errores positivos se asociaron a la expansión económica estadounidense.

Por otra parte, al observar los errores en el corto y en el largo plazo existen diferencias entre la propensión marginal al consumo a corto y largo plazo. Según Hall y Taylor (1988), la propensión marginal en el largo plazo es mayor que en el corto plazo, tanto para bienes durables como para no durables. Si se produce una situación como la descrita entonces se puede concluir que la teoría del consumo de Keynes es falsada, ya que los errores son expresión de que los datos reales no se

\footnotetext{
16 Según John Maynard Keynes: "Por consiguiente, definiremos lo que hemos llamado la propensión a consumir como la relación funcional X entre Yn, un nivel de ingreso dado, medido en unidades de salario, y $\mathrm{Cn}$, el gasto que para el consumo se toma dicho nivel de ingreso, de manera que $\mathrm{Cn}=\mathrm{X}$ (Yn) o S = S.X (Yn)" (Keynes, 1981: 88).
} 
ajustan a la función descrita de consumo agregado.

Frente a esta situación, algunos economistas se negaron a descartar $\mathrm{Yd} \rightarrow \mathrm{C}$. El consumo no es simplemente destinar dinero para consumir, envuelve una decisión del consumidor y esa decisión tiene una serie de consideraciones. Eso llevó a científicos en las ciencias naturales y en la Economía a proponer hipótesis "ad hoc", que son argumentos adicionales para explicar las contradicciones "aparentes" entre las predicciones y las observaciones ${ }^{17}$.

De esta manera, según Hall y Taylor (1988), se presentan varias hipótesis ad hoc que tratan de mejorar la perspectiva de Keynes: la hipótesis del ciclo de vida de Modigliani y Ando (1963); la hipótesis del ingreso permanente de Milton Friedman (1970); las expectativas racionales, incluyendo la hipótesis de paseo aleatorio (random walk); las historias de familias individuales (individual family histories)

\footnotetext{
${ }^{17}$ En este sentido no formuló hipótesis "ad hoc", pero de alguna manera sentó ideas para propuestas más formalizadas en el futuro cuando expresó: "[l]a suma que la comunidad gasta en el consumo depende evidentemente de 1) el monto de su ingreso, 2) otras circunstancias objetivas que lo acompañan, y 3) las necesidades subjetivas y las inclinaciones psicológicas y hábitos de los individuos, así como los principios según los cuales se divide el ingreso entre ellos" (Keynes, 1981: 88).
}

o los experimentos de políticas económicas (policy experiments).

En el caso de Modigliani y Ando, el consumo depende de dos factores: 1) el ingreso corriente del trabajo, y 2) los activos. Así, dado un nivel de activos, un cambio en el ingreso se asume como un cambio en el ingreso a largo plazo, por lo que la propensión al consumo se acercará a 1 . En el caso de Friedman, el consumo dependerá del denominado ingreso permanente (permanent income) Yp, que es un promedio histórico de los ingresos de las personas. En la hipótesis de paseo aleatorio, el consumo sigue un sendero aleatorio si la mejor creencia de la variable en el próximo periodo es la misma que en el periodo anterior. Por otra parte, universidades como la de Michigan establecieron el llamado Panel de estudio sobre dinámicas del ingreso (Panel Study on Income Dynamics) en 1969 para conocer las experiencias de las familias en situación de auge y de recesión económica. Finalmente, los gobiernos (por ejemplo, Lyndon B. Johnson o Gerard Ford) han aplicado medidas impositivas para ajustar o expandir la demanda agregada, lo que le ha dado a los economistas la oportunidad de probar las teorías intertemporales del consumo.

La presencia de hipótesis ad hoc alimentó planteamientos como la famosa te- 
sis de Duhem-Quine, que según Lakatos consiste en que: "[c]ualquier teoría puede ser permanentemente salvada de la refutación mediante ajustes adecuados en el conocimiento básico en el que se halla inserta" (1998: 127). Es decir, una teoría no puede ser completamente refutada, debido a que los exámenes dependen de elementos que escapan al control de las teorías. Es decir, que en Economía la teoría del consumo keynesiana no puede ser completamente falsada.

Con Lakatos (1998), Feyerabend (1989) y Kuhn (2004) y se abre una crítica al realismo crítico de Popper, ya que no existen hechos puros en la observación y el cuestionamiento de la posibilidad de axiomatizar las ciencias empíricas (Cuadrado y Valmayor, 1999). Para Lakatos, los investigadores sustituyen una teoría cuando la nueva teoría presenta un mayor contenido empírico que le permite superar enigmas, anomalías y hacer mejores predicciones. En consecuencia, los "programas de investigación" (scientific research programmes) son cadenas de teorías relacionadas que se encuentran sometidas a la evaluación mediante confirmaciones continuadas. Esas teorías cuentan con un núcleo fuerte y estable (con la teoría principal) y un conjunto de proposiciones periféricas que se exponen a modificaciones.
En Economía, un ejemplo de lo que Lakatos propuso se puede exponer con lo que le sucedió a la teoría clásica (principalmente Arthur Cecil Pigou, Alfred Marshall o Knut Wicksell en la terminología de Keynes) y el esquema de demanda efectiva de John Maynard Keynes. El esquema de demanda efectiva mantiene supuestos del modelo clásico de propiedad privada, cálculo racional de los agentes económicos, los gustos constantes, la independencia de los agentes económicos en sus decisiones, entre otros supuestos. No obstante, critica las ideas periféricas tales como la equivalencia entre el salario y la productividad marginal del trabajo, la imperfecta movilidad de factores, los ajustes no automáticos e instantáneos de la oferta y demanda agregada. Más aún, el propio Keynes apunta que la teoría clásica es un caso particular de la demanda efectiva:

"La demanda efectiva que trae consigo la plena ocupación es un caso especial que sólo se realiza cuando la propensión a consumir y el incentivo para invertir se encuentran en una relación mutua particular. Esta relación particular, que corresponde a los supuestos de la teoría clásica, es, en cierto sentido, una relación óptima; pero sólo puede darse cuando, por accidente o por designio, la inversión corriente provea un volumen de demanda justamente igual al excedente 
del precio de la oferta global de la producción resultante de la ocupación plena, sobre lo que la comunidad decidirá gastar en consumo cuando la ocupación se encuentre en ese estado" (1981: 36).

Para Feyerabend: "[l]a idea de un método que contenga principios científicos inalterables y absolutamente obligatorios que rijan los asuntos científicos entra en dificultad al ser confrontada con los resultados de la investigación histórica" (1989: 14). La Economía como ciencia histórica debe tomar nota de este problema.

Por otra parte, para Kuhn (2004) existe en la comunidad científica lo que él denomina como ciencia normal y ciencia extraordinaria. La primera se sustenta en una o más realizaciones científicas y métodos que son reconocidos como legítimos. La ciencia extraordinaria hace referencia a las comunidades científicas que surgen alrededor de un nuevo paradigma. La ciencia normal constantemente se encuentra desafiada por nuevos descubrimientos, la presencia de enigmas y la existencia de anomalías. Esas anomalías se tratan de ajustar a la ciencia normal, pero en el proceso surgen nuevas formas de explicación que compiten con las ideas establecidas y se produce una competencia entre paradigmas. Ahora bien, el triunfo del nuevo paradigma se presenta cuando resuelve anomalías y enigmas ${ }^{18}$. Sin embargo, el triunfo del nuevo paradigma no implica su aceptación por el resto de la comunidad científica. Esta aceptación implica una lucha y una reconstrucción del campo o la disciplina. Una vez legitimado el nuevo paradigma se convierte en ciencia normal.

En Economía un paradigma legitimado, no significa que sea hegemónico ni siquiera en los problemas y métodos en que se considera fuerte. En Economía para un mismo problema hay diferentes posturas que polemizan, porque responden generalmente a posiciones filosóficas o corrientes ideológicas. Esto nos lleva a la idea de que el paradigma más "popular" convive con posturas críticas. Por esta razón, se debería hablar de las tradiciones de investigación de Larry Laudan (1978). Laudan propone, entre otras cosas, dos ideas: 1) que la función de la ciencia es resolver problemas o responder de forma

\footnotetext{
${ }^{18}$ Para Kuhn, como los paradigmas son irreconciliables, la sustitución de un paradigma viejo por otro nuevo es casi total. Este cambio implica una nueva visión del mundo. Por último, se puede destacar que Kuhn (2004) niega que la realidad estudiada por los científicos, entre ellas la económica, sea independiente de los paradigmas que ellos usan y "[r]echaza que la ciencia crezca mediante acumulación de verdades eternas" Lakatos (1998:18). Esta propuesta no permite hablar de verdad como correspondencia o adecuación entre la ontología de una teoría y lo que realmente hay.
} 
aceptable a preguntas interesantes; y 2) la idea de tradiciones de investigación (research traditions). Para Laudan (1978), la ciencia es una actividad pragmática que no busca establecer la verdad o falsedad de proposiciones y que, por tanto, su desarrollo en el tiempo es discontinuo y no acumulativo.

En otras palabras, el progreso científico se presenta cuando una teoría que compite con otras teorías posee una mayor capacidad para resolver problemas importantes. Los problemas para Laudan (1978) se dividen en teóricos y empíricos. Los problemas empíricos se dividen, a su vez, en potenciales (esperando por ser resueltos), resueltos y anómalos (aquellos problemas no resueltos en una teoría pero sí resueltos en otra dentro de la tradición de investigación). El progreso de la ciencia va por el camino de que con datos empíricos una teoría no sólo ofrezca una solución más ventajosa, sino que también tenga un mejor poder explicativo.

Por otra parte, este autor introduce el concepto de tradición de investigación (por ejemplo, en la física la teoría electromagnética de la luz y, en el caso de la economía, el keynesianismo o el marxismo). Esta es una comunidad científica en donde conviven diferentes perspectivas a la hora de abordar problemas cruciales de la ciencia. Diferentes teorías, dentro de la tradición, tienen explicaciones diferentes que luchan por la interpretación más aceptada en términos pragmáticos $\mathrm{y}$, generalmente, tienen un largo historial.

En Economía, por ejemplo, se tendría la tradición económica keynesiana que comenzó con: 1) los denominados keynesianos (Roy Harrod, Evsey Domar o Alvin Hansen), quienes aplicaron la General Theory de J. M. Keynes (diseño de modelos de crecimiento con multiplicador y acelerador); 2) los economistas que combinaron aspectos de la tradición neoclásica con el keynesianismo y culminó con la famosa síntesis neoclásica promovida por John Hicks, Paul Samuelson y Franco Modigliani entre otros; 3) los reinterpretadores de la General Theory tales como Michal Kalecki, Piero Sraffa, Luigi Pasinetti, Joan Robinson Robert Clower o Axel Leijonhufvud, quienes son críticos de la síntesis neoclásica; 4) los PostKeynesianos tales como Nicholas Kaldor, George Lennox Sharman Shackle, Paul Davidson, Edmond Malinvaud, Sidney Weintraub o Hyman Misnky, quienes introdujeron el marco político-institucional en el análisis macroeconómico; y 5) la nueva economía keynesiana (con autores tales como Olivier Blanchard, Gregory Mankiw, Frederic Mishkin o Joseph Stiglitz), con la que se emplea el marco de 
la competencia imperfecta, la existencia de rigideces de precios y salarios y las decisiones de los agentes económicos que no se ajustan al esquema walrasiano de oferta y demanda.

Dentro de estas diferentes tendencias de la tradición keynesiana prevaleció la síntesis neoclásica, al punto que se convirtió en la corriente principal o mainstream $^{19}$. Su surgimiento se ubica después de la Segunda Guerra Mundial y recoge los aportes macroeconómicos keynesianos comprendidos en el estudio de la demanda agregada y los aportes neoclásicos más enfocados del lado microeconómico y la oferta.

\footnotetext{
19 La denominada corriente principal, hegemónica o dominante como algunos economistas las califican no es en sí una escuela. Son en realidad grupos de economistas que se agrupaban en dos conjuntos de universidades estadounidenses denominadas Escuela de agua dulce y Escuela de agua salada (Freshwater School y Saltwater School). La primera corresponde a universidades ubicadas principalmente en Estados de la unión americana con costas en los Grandes Lagos como la Universidad de Minnesota, Universidad de Chicago, Universidad de Rochester, Universidad de Pittsburgh y Universidad Carnegie Mellon. Las universidades del segundo grupo correspondían a Harvard, Berkeley, MIT, Universidad de Pensilvania, Universidad de Stanford y la Universidad Yale. Las tradiciones de pensamiento económico que no se encuentra dentro del mainstream son, por ejemplo, la economía austríaca, el institucionalismo, la economía marxista, la economía social de mercado, la economía feminista, el socialismo económico, la economía ecológica, entre otras tradiciones.
}

La síntesis neoclásica sigue en líneas generales lo siguiente: 1) la relación medio-fin planteada en su momento por Robbins (1980); 2) acepta la idea de costo de oportunidad; 3) acepta el individualismo metodológico bajo el término de "agente representativo"; 4) acepta las posturas de la teoría de la elección racional; 5) acepta que los mercados son imperfectos, que existe asimetría de información, información incompleta, competencia imperfecta y la existencia de costos de transacción; 6) metodológicamente emplean el lenguaje matemático mediante el diseño de modelos macroeconómicos como el modelo ISLM (Investment Saving- Liquidity Preference Money Supply), que fue presentado por John Hicks en 1937; o 7) que la acción política no debe ser empleada para resolver problemas del sistema económico sino que debe ser producto de la acción racional de los agentes económicos.

La crisis económica que estalló en 2007 es una prueba de que en la ciencia económica conviven tradiciones de investigación que en algún momento del tiempo coinciden en sus planteamientos generales y en ocasiones no. En este caso, el conflicto se centró, por ejemplo, en que un grupo de economistas, denominados 
Chartalistas $^{20}$, consideraron que para tener una comprensión de las cusas de la crisis económica es necesario dar cuenta de cómo el dinero realmente fluye desde su emisión por parte de las autoridades con capacidad legal, el sistema financiero (mercados de intermediarios bancarios, mercado de dinero, de capitales y cambiario) y los diferentes sectores de la economía. Estas consideraciones implicaron que estos economistas rechazaran la idea del mainstream de los mercados de fondos

\footnotetext{
${ }^{20}$ Es una teoría del dinero que enfatiza el impacto de la política monetaria del gobierno sobre el valor del dinero y las actividades económicas. Su promotor inicial fue el economista alemán Georg Friedrich Knapp. Esta teoría parte de la idea de que el dinero es la unidad de cuenta cuyo valor no está determinado de manera intrínseca, sino que su valor proviene de los que el gobierno espera de él. El término Chartalismo (Chartalism en inglés) proviene del Latin "charta" que significa ticket o recibo. Actualmente sus seguidores se les denomina seguidores de la teoría moderna del dinero (Modern Money Theory MMT o Neochartalism). Esta teoría busca detallar los procesos y consecuencias del uso por parte del Gobierno del dinero bajo la forma de dinero fiduciario (Fiat pecūnia). Según esta teoría, el dinero es establecido y creado por el Gobierno y entra en circulación a través del gasto público o los impuestos. En este sentido la MMT mantiene la idea de que el nivel de impuestos con relación al gasto público es en realidad una herramienta de política económica que busca regular la inflación y mantener bajo el desempleo y no un medio para fundamentar sus actividades per se. Entre sus modernos proponentes se encuentran Warren Mosler, Bill Mitchell, Stephanie Kelton y Randall Wray. Sus principales críticos provienen del mundo post y nuevo-Keynesiano, concretamente de Paul Krugman y de la escuela austríaca, principalmente de la mano de Robert P. Murphy.
}

prestables, el multiplicador monetario $\mathrm{y}$ la efectividad de las políticas fiscales de austeridad.

Otra de las controversias surgidas a lo largo de los años, concretamente en el análisis monetario, se ha desarrollado a partir de estudios de los cuales se ha querido ofrecer evidencia empírica de la relación de las cantidades de dinero que se generan en una economía y, por ejemplo, el producto interno bruto. En algunos estudios se ha puesto en evidencia la importancia del dinero sobre las variables macroeconómicas y en otros no es tan evidente esa importancia.

La controversia entre quienes apoyan la tesis de los efectos del dinero en la actividad económica y quienes no lo sustentan ha alimentado la controversia entre dos tipos de evidencia empírica que son afines a dos posturas en la ciencia económica, a saber, los monetaristas y los keynesianos (Mishkin, 1995). Por un lado se encuentra la evidencia basada en modelos estructurales (structural model evidence), que analiza si una variable o un grupo de variables afectan a otras variables por medio del uso de información o data para construir modelos que tratan de exponer los canales a través de los cuales permita dar cuenta de la relación del dinero con la actividad económica. Por otra parte, 
se encuentran los modelos que emplean evidencias de la forma reducida (reducedform evidence), que examinan si una variable tiene un efecto sobre otra variable observando, por ejemplo, directamente la relación entre el comportamiento de la actividad económica y el comportamiento de la oferta de dinero.

En los modelos estructurales, los efectos del dinero sobre la actividad económica se estudian construyendo sistemas de ecuaciones que representan sectores de la economía que describen cuáles son los canales por medio de los cuales la política monetaria y fiscal afecta la actividad económica. El resultado del estudio es la configuración de los canales de transmisión de la política monetaria. Por su parte, la evidencia mediante la forma reducida, no busca describir un canal específico de transmisión. En su lugar, estudia la correlación entre el dinero en la actividad económica.

\section{Las tradiciones de investigación y el realismo}

A propósito de las dos controversias analizadas anteriormente, en la década de los años 60 del siglo $\mathrm{XX}$ resurge la idea de que la filosofía de la ciencia debe prescribir criterios para que las teorías alcancen la verdad de una manera razonada. Surge el realismo con las siguientes propuestas: 1) que el mundo estudiado por la ciencia es independiente de la actividad de la conciencia cognoscente; 2) la ciencia es la mejor forma de dar cuenta de la realidad; 3) revela entidades no observables del mundo y explica sus propiedades; y 4) las leyes científicas aparecen como premisas inevitables y verdaderas acerca del mundo.

Por otra parte, surge el antirrealismo que propone, en la versiones de Duhem (1969) y Bas van Frassen (1996): 1) establecer relaciones entre observaciones que exhiben apariencias sensibles; 2) emitir sentencias estableciendo relaciones entre observaciones para proponer nuevas relaciones; 3) las leyes no son verdaderas ni falsas, sólo instrumentos de predicción ; 4) la ciencia sólo se interesa por la predicción; y 5) lograr teorías "empíricamente adecuadas" que permitan alcanzar la verdad sólo acerca de observables para "salvar las apariencias".

De la crítica al antirrealismo de Bas van Frassen (1996), surgieron una serie de realismos disminuidos. Fine (1996) declara su aceptación del conocimiento del sentido común porque es confiable sin que esto conduzca a un progreso de la ciencia. Cartwright (1984) plantea la diferencia entre leyes fundamentales (como la mecá- 
nica clásica) y las leyes fenomenológicas (aproximaciones sucesivas en el comportamiento de los cuerpos reales). Cuando de una ley fenomenológica es deducida una fundamental y los hechos de los que da cuenta son verdaderos, se puede hablar de verdad de las causas operantes. El realismo interno de Putnam (1988) parte de un marco conceptual que establece el número, significado y propiedades de los objetos que son aceptados. Los marcos conceptuales son aceptados, pero sin ser verdades absolutas. Además, las condiciones de afirmación se aprenden con la práctica y no pueden ser totalmente formalizadas.

Desde la perspectiva de Searle, es de gran importancia lo que denominó como la "tesis de la relatividad conceptual" (Searle 1997:160). Esta tesis hace referencia a la posibilidad de la existencia de un número indefinidamente grande de diversos sistemas de representación para dar cuenta de las experiencias. Considera que cualquier sistema de denominación o de clasificación para describir el mundo es convencional. Según Searle (1997), no existe una respuesta absoluta y ésta dependerá de la elección convencional de esquemas.

Para el realismo perspectivista (constructivo) de corte naturalista de Giere (1999), la realidad es estudiada por la cien- cia mediante modelos que son representaciones aproximadas. Por tanto, la verdad de un enunciado estará en función de la "similaridad" entre el modelo conformado por enunciados y aquello de lo que trata de dar cuenta. Se denomina perspectivista porque se plantean modelos que crecen en sofisticación representativa, y es de corte naturalista en el sentido de que diferentes modelos compiten por alcanzar un mayor grado de adecuación con los hechos. Por otra parte, el naturalismo se entiende como un modelo teórico o representación primaria de los objetos en la ciencia; tales objetos son, según Giere: "objetos abstractos, entidades imaginarias en las cuales sus estructuras pueden ser o no similares a determinados aspectos de los objetos y procesos en el mundo real" (1999:5).

Para este autor, las denominadas leyes de la naturaleza son ambigüedades y más bien puede hablarse de principios, que constituyen reglas generales que ayudan a la construcción de modelos. Dentro del modelo, por lo tanto, las proposiciones son verdaderas; sin embargo, no son proposiciones que den cuenta del mundo. Con respecto al aspecto empírico, Giere sugiere que: "el ajuste entre la proposición y el denominado mundo real es parcial e imperfecto. No hay tal cosa como un modelo perfecto, completo en todos sus detalles" (Giere, 1999:6). Es decir, entre la repre- 
sentación (mapa) y el denominado mundo real puede ser construida socialmente, pero expresa que también se debe aceptar que las representaciones socialmente construidas pueden ser descubiertas con ayuda del método científico, con la finalidad de ofrecer un buen cuadro de determinados aspectos del mundo (Giere, 2004). El ajuste adecuado se dará por medio de las "decisiones cruciales", en que las afirmaciones son construidas por grupos de personas con competencias para realizar juicios acerca de los fenómenos objeto de estudio.

En consecuencia, desde el punto de vista de Giere, el realismo perspectivista se distancia tanto del empirismo lógico como del relativismo sociológico; ambos, según el autor, de una u otra manera, evaden el rol genuino de las representaciones como imagen de la ciencia. En lo que respecta al trabajo de Nancy Cartwright (1994), las teorías expresan verdades acerca de las "tendencias" y "capacidades" que subyacen en los fenómenos; es decir, la validez es contextual y depende de propiedades causales intrínsecas de los sistemas sometidos a evaluación. Según Cartwright (1999), para que en dos contextos los resultados de una correlación o regresión sean semejantes a lo que propone una teoría, el sistema debe estar altamente estructurado, con objetos dotados de capacidades que permiten la obtención de correlaciones estadísticas nomológicas significativas entre sus valores observables.

\section{Desafíos del análisis macroeconómico}

Al admitir como interesante la idea de tradiciones de investigación, se puede hablar de una tradición marxista, una tradición neoclásica, el institucionalismo o una tradición keynesiana. Dentro de estas tradiciones surgen propuestas para dar cuenta de la realidad de los problemas económicos. Estas propuestas en algunos casos son convergentes, en otros complementarios o divergentes. Un ejemplo son el operacionalismo de Samuelson y el instrumentalismo de Friedman, o entre los nuevos keynesianos y la denominada nueva teoría monetaria. La Economía tiene como desafío introducir en el análisis la agenda política, que responde significativamente a la lógica de maximizar los apoyos o minimizar los abandonos. En una sociedad democrática con criterios de elección mayoritaria, tal vez desde el punto de vista de determinada tradición de investigación, un determinado problema económico es factible de solución, pero se enfrenta con la viabilidad política (costo político en términos de rechazo o abandono de los apoyos). 
Por otra parte, se debe indicar que no es igual un problema económico, que cuenta con un alto componente de magnitudes sociales, políticas o antropológicas, que resolver un sistema de ecuaciones compatibles, en donde todos sus componentes se forman con cantidades económicas. La pregunta que surge es ¿hasta qué medida, de ser posible, un problema económico, por medio de modelos y recomendaciones de política económica, resuelve lo que enuncia que debe ser resuelto desde una perspectiva intertemporal?

Además de lo antes expuesto, la ciencia económica debe: 1) luchar para que su léxico sea cada vez menos oscuro y opaco; 2) sin buscar ser historicista, la Economía no debe olvidar que el fenómeno económico tiene un fuerte referente histórico y espacial, lo que Braudel denominó como "Geohistoria" (Burke, 1993); 4) se debe tomar en cuenta que la sociedad es una pluralidad de campus y habitus en el sentido de Bourdieu (2001), es decir, estructuras que se organizan en función de sus respectivos capitales simbólicos, económicos y culturales y que establecen reglas de entrada, permanencia y promoción para los diferentes miembros que intervienen en él de manera conflictiva.

\section{Conclusiones}

De acuerdo con lo antes expuesto, la ciencia económica no busca verificar o corroborar enunciados. La ciencia económica se aproxima más a los planteamientos realistas en una versión perspectivista de corte naturalista; por cuanto los modelos económicos, constituyen una herramienta de estudio que permiten dar cuenta, aunque sea de forma parcial, de fenómenos económicos de interés para los que diseñan e implementan políticas económicas.

Por otra parte, el carácter imperfecto de los modelos macroeconómicos y la presión por parte de los diseñadores y ejecutantes de políticas públicas económicas han llevado a que el conocimiento económico tienda principalmente a la solución de problemas económicos. Desde este punto de vista y siguiendo a Laudan (1978), el progreso de la Economía estará en su capacidad para resolver un mayor número de problemas económicos. Pero siendo la Economía, desde este punto de vista, una disciplina preferentemente pragmática no implica que se deba interesar en la ciencia económica per se, en la comprensión del fenómeno económico. 
A las tensiones de carácter epistemológico hay que agregar las tensiones de carácter político y social provenientes de los diferentes actores sociales (tales como el público, los partidos políticos o, por ejemplo, fundaciones privadas nacionales o internacionales, encargadas de hacer circular representaciones de ciertas ideas económicas). En otras palabras, el conocimiento teórico, aunque implique el uso de referentes conceptuales y empíricos adecuados en los términos antes expuestos, se enfrentan con el problema de la viabilidad político-social y la crítica desde las diferentes esferas de poder. Estas consideraciones deberían llevar a la teoría Económica a afrontar una tensión entre propuestas teóricas con mayor capacidad de resolver problemas, y que esas propuestas aceptadas sean, de alguna manera, pertinentes o moralmente valiosas para la sociedad para evitar convertir las propuestas teóricas en metáforas (McCloskey, 1983) y convertir el discurso económico en una forma de retórica persuasiva.

\section{Referencias}

Ayer, A. (1981). El positivismo lógico. México: FCE.
Bachelard, G. (1997). La formación del espíritu científico. Contribución al psicoanálisis delo conocmiento objetivo. México, D. F.: Siglo Veintiuno.

Blaug, M. (1993). La metodología de la economía. Madrid: Alianza.

Bourdieu, P. (2001). Poder, Derecho y clases sociales. Segunda edición. Bilbao: Desclée de Brouwer.

Bridgman, P. (1927). Thelogic of modern physics. New York: McMillan.

Bunge, M. (1980). Epistemología. Barcelona: Ariel.

Bunge, M. (1989). La investigación cientifica. Barcelona: Ariel.

Burke, P. (1993). La revolución historiagráfica francesa. La escuela de los Annales: 1929-1989. Primera edición. Barcelona: Gedisa.

Cairnes, J. (2001). The character and logical method of political economy. Kitchener: Batoche Books.

Carnap, R. (1992). Testability and meaning. En: H. Feigl y M. Brodbeck. Readings in the philosophy of science. (47-92). Minneapolis: University of Minnesota Press.

Cartwright, N. (1984). How the Laws of Physics Lie. London: Clarendon Press. 
Cartwright, N. (1994). Nature's capacities and their measurement. London: $\mathrm{Ox}-$ ford University Press.

Cartwright, N. (1999). The dappled world: A study of the boundaries of science. Cambridge: Cambridge University Press.

Cuadrado, A. y Valmayor, M. (1999). Teoría contable. Metodología de la investigación contable. Madrid: McGraw Hill.

Duhem, P. (1969). To save the phenomena: An essay on the idea of physical theory from Plato to Galileo. Chicago: The University of Chicago Press.

Ferguson, C. E. y Gould, J. P. (1979). Teoría microeconomíca. Madrid: Fondo de Cultura Económica.

Ferguson, J. M. (1979). Historia de la Economía. México, D. F.: Fondo de Cultura Económica.

Feyerabend, P. (1989). Contra el método. Esquema de una teoría anarquista del conocimiento. Barcelona: Ariel.

Fine, A. (1996). The shaky game: Einstein, realism and the quantum theory. Chicago: The University of Chicago Press.
Frey, B. (2000). Why Economist Disregard Economic Methodology. Journal of Economic Methodology, 8(1): 41-47.

Friedman, M. (1967). Ensayos sobre economía positiva. Madrid: Gredos.

Friedman, M. (1970). A theory of the consumption function. New Delhi: Oxford.

Giere, R. (1999). Science without laws. Chicago: The University of Chicago Press.

Giere, R. (2004). How models are used to represent reality. Philosophy of Science, 71(5): 742-752.

Hall, R. y Taylor, J. (1988). Macroeconomics: Theory, performance, and policy. New York: W. W. Norton.

Hanson, N. (1972). Patterns of discovery: An inquiry into the conceptual foundations of science. Cambridge: Cambridge University Press.

Hempel, C. (1981). Problemas y cambios en el criterio empirista del significado. En: A. Ayer. El positivismo lógico. (115-137). México: FCE.

Hempel, C. (2006). La explicación cientifica. Estudios sobre la filosofía de la ciencia. Buenos Aires: Paidós. 
Keynes, J. (1981). Teoría general de la ocupación, el interés y el dinero. México: FCE.

Keynes, J. N. (1990). The scope and Method of Political Economy. Kitchener: Batoche Books.

Kuhn, T. (2004). La estructura de las revoluciones científicas. Buenos Aires: FCE.

Lakatos, I. (1998). La metodología de los programas de investigación científica. Madrid: Alianza.

Laudan, L. (1978). Progress and its problems: Towards a theory of scientific growth. Berkeley: University of California Press.

Mays, W. (1962). Jevons's Conception of Scientific Method. The Manchester School, 30(3): 223-249.

McCloskey, D. (1983). The rhetoric of economics. Journal of Economic Literature, 21(2): 481-517.

McCloskey, D. (1997). The Vices of Economists; The Virtues of the Bourgeoisie. Amsterdam: University of Amsterdam Press.

McCloskey, D. y Ziliak, S. (1996). The Standard Error of Regression. Journal of Economic Literature, 34: 97114.
Menger, C. (2006). El método de las ciencias sociales. Madrid: Unión Editorial.

Mill, J. (1874). Essays on Some Unsettled Questions of Political Economy. London: Longmans, Green, Reader, and Dyer.

Mishkin, F. (1995). The economics of money, banking, and financial markets. New York: Harper Collins Publishers.

Modigliani, F. y Ando, A. (1963). The 'life-cycle' hypothesis of saving: Aggregate implication and test. American Economic Association, 53(1): 55-84.

Munt, J. y Barrionuevo, G. (2010). Reflexiones sobre la ontología de la economía: La visión tradicional vs. la heterodoxia moderna. Kairos, 14(26).

Popper, K. (1980). La Lógica de la investigación científica. Madrid: Tecnos.

Popper, K. (1991). Conjeturas y refutaciones. El desarrollo del conocimiento científico. Buenos Aires: Paidós.

Putnam, H. (1988). Representation and reality. Boston: MIT Press.

Rima, I. (1995). Desarrollo del Análisis Económico. Madrid: Elsevier.

Robbins, L. (1980). Ensayo sobre la naturaleza y significación de la ciencia económica. México, D. F.: Fondo de Cultura económica de México. 
Sacristán, M. (1978). El trabajo científico de Marx y su noción de ciencia. Recuperado de http://archivo.juventudes.org/textos/Manuel\%20Sacristan/El\%20trabajo\%20cientifico $\% 20$ de\%20Marx\%20y\%20su\%20nocion\%20de\%20ciencia.pdf.

Schumpeter, J. (1994). Historia del Análisis Económico. Barcelona: Ariel.

Searle, J. (1997). La construcción de la realidad social. Barcelona: Paidós.

Senior, N. (1854). Political Economy. London: J. Mawman.

Shackle, G. (1976). Epistémica y economía: crítica a las doctrinas económicas. Madrid: FCE.
Sidgwick, H. (1901). The principles of Political Economy. London: Macmillan and Co., Limited.

Toulmin, S. (1953). The philosophy of science: An introduction. London: Hutchinson's University Library.

Van Frassen, B. (1996). La imagen científica. Buenos Aires: Paidós.

Wittgenstein, L. (2003). Tractactus Logico-Philosophicus. Madrid: Alianza Editorial.

Zamora, F. (1977). Tratado de teoría económica. México, D. F.: Fondo de Cultura Económica. 